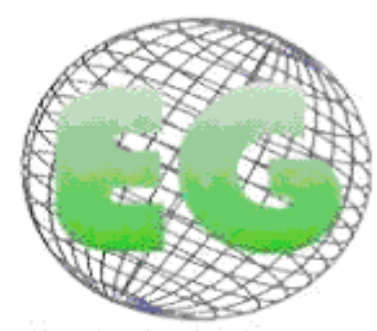

ISSN 1695-6141 N 27

\title{
Aproximación teórica del profesional enfermero a los dispositivos legales para afrontar la violencia de género
}

Theoretical professional nurssing approach to the legal devices state gives to face gender violence

\author{
*Carrilero López, S., *Martínez Díaz, JM., "*Flores Bienert, MD. \\ *Enfermera. E-mail: juanamaria.martinez6@um.es **Doctora en Educación. Profesora de la Facultad de \\ Enfermería de la Universidad de Murcia.
}

Palabras clave: enfermería; violencia contra la mujer; derechos de la mujer.

Keywords: nursing; violence against women; women's rights.

\section{RESUMEN}

La violencia contra la mujer ha constituido un fenómeno invisible durante décadas. Incluso hoy continúa siendo difícil de identificar. Así, la II Conferencia Mundial sobre la Condición Jurídica y Social de la Mujer celebrada en 1980 en Copenhague ya se refería a este tipo de maltrato como "el crimen más silenciado del mundo".

Nosotros, como enfermeros guiados por conocimientos legales, éticos y deontológicos, y junto a otros profesionales, nos percatamos de la inmensa importancia que tiene la defensa de los derechos humanos y por ello creemos oportuna la realización de este estudio en el que intentaremos resumir los distintos sistemas de protección estatales para satisfacer nuestro objetivo último: acercar al profesional enfermero que lea estas líneas, al conocimiento de dichos sistemas y al contexto de la violencia de género.

La técnica empleada en esta revisión bibliográfica es la de análisis de contenido, en la modalidad temática, que se basa en la lectura como instrumento de recogida de datos; lectura que debe realizarse siguiendo el método científico, es decir, debe ser sistemática, objetiva, replicable y válida.

Todos estos dispositivos de protección estatales son relevantes pero no debemos de olvidar uno en absoluto desdeñable: el equipo interdisciplinar sanitario entre el que se incluye el conjunto de Enfermería que, al conocer dichos dispositivos, puede convertirse en un aliado destacado a la hora de garantizar la seguridad e integridad de la mujer.

\section{ABSTRACT}

Violence against women has been an unseen phenomenon for decades. Even today it remains being difficult to identify. Thus, In the Second World Conference about the Status of women celebrated in Copenhagen in 1980 referred this type of abuse as "the most silenced crime in the world."

We, as nurses guided by legal, ethical and deontological knowledge, and with other professionals, notice the immense importance of defending the Human Rights and because of that, this issue is the reason for this study 
in which we try to summarize the various State Protection Systems in order to get our ultimate goal: to approach the professional nurse who reads these lines to the knowledge of these systems and the knowledge of the context of gender violence.

The technique used in this review is a content analysis procedure, within its thematic category which is based on reading as a tool of data collection; reading that has to be done following the scientific method, i.e. it must be systematic, objective and valid.

All these State Protective devices are relevant, but we must remember one important aspect: the interdisciplinary health team which includes among the set of nursing that, realizing of this devices, can become in a prominent ally in women's ensuring security and integrity.

\section{INTRODUCCIÓN}

La violencia contra las mujeres es, en la actualidad, un tema de preocupación social en el que los medios de comunicación han volcado una gran atención. Este hecho puede hacer parecer que los malos tratos están en boga y que van en aumento. Pero en realidad, la violencia y los malos tratos han formado parte de nuestra vida cotidiana a lo largo de la historia, aunque al estar normalizada y naturalizada pasaban desapercibidos: la violencia contra la mujer estaba silenciada y oculta. Basamos estas consideraciones en la II Conferencia Mundial sobre la Condición Jurídica y Social de la Mujer celebrada en 1980 en la ciudad de Copenhague donde ya se refería a este tipo de malos tratos como "el crimen más silenciado del mundo" (1)

En los últimos diez lustros la posición de la mujer no solamente en España, sino en el mundo en general se ha transformado, siendo muchos los factores que han participado en tal cambio: avances científicos, desarrollo de medidas de planificación familiar, aumento de la concepción de la mujer como sujeto de derechos, mayor facilidad de acceso a la formación en todos los niveles, avance en la legislación referida al tema ${ }^{(2)}$. De la misma forma la impunidad y el poder social para resolver con violencia los problemas dentro de la familia por los varones han sido cuestionados y han ido decreciendo. Sin embargo, esta concepción no se haya erradicada por completo ya que todavía existen hombres violentosque buscan mujeres que puedan ser dominadas y que ven en la agresión y en el insulto la única solución válida para cumplir su propósito cuando la mujer "no cumple con sus expectativas". Estas sospechas están avaladas por distintos datos estadísticos. A nivel mundial, el $68 \%$ de las mujeres asesinadas mueren a manos de sus parejas, maridos o exparejas. ${ }^{(3)}$

Asimismo, es importante determinar la repercusión que esta situación conlleva en nuestro país. Según datos del Instituto Nacional de Estadística y del Ministerio de Sanidad, Política Social e Igualdad, durante el año 2010 se produjeron 73 muertes de mujeres a manos de sus parejas, 18 más que las ocurridas durante el año anterior. En este año, 2011 y a fecha 28 de noviembre (tres días después del día Internacional para la Eliminación de la Violencia contra la Mujer y un día después de la última muerte perpetrada en Terrasa, Barcelona), se ha contabilizado un total de 55 víctimas. En la tabla I se presenta el número de muertes por violencia contra la mujer en distintos años. En la tabla II se muestra la distribución por edad de las víctimas mortales producidas en el año 2011 . $^{(4,5)}$ 
Tabla I: Víctimas mortales por violencia de género.

$\begin{array}{llllllllllll}2000 & 2001 & 2002 & 2003 & 2004 & 2005 & 2006 & 2007 & 2008 & 2009 & 2010 & 2011 \\ 63 & 50 & 54 & 71 & 72 & 57 & 68 & 71 & 76 & 55 & 73 & 55^{*}\end{array}$

${ }^{*}$ A fecha de 28 de noviembre.

Fuente: 2000-2010: Elaborado por INE a partir de noticias de prensa y datos del Ministerio del Interior. A partir de 2006 datos de la Delegación del Gobierno contra la Violencia de Género. ${ }^{(6)}$

Nota: Se incluyen aquellos casos en los que el agresor es el cónyuge, excónyuge, compañero sentimental, excompañero sentimental, novio o exnovio.

Tabla II. Víctimas mortales por violencia de género según edad.

\begin{tabular}{|c|c|c|}
\hline & $\begin{array}{l}\text { Número de } \\
\text { casos }\end{array}$ & $\begin{array}{l}\text { Porcentaje } \\
(\%)\end{array}$ \\
\hline Menos de 16 & 0 & 0 \\
\hline De 16 a 17 & 0 & 0 \\
\hline De 18 a 20 & 3 & 5,5 \\
\hline De 21 a 30 & 13 & 23,6 \\
\hline De 31 a 40 & 14 & 25,5 \\
\hline De 41 a 50 & 12 & 21,8 \\
\hline De 51 a 64 & 6 & 10,9 \\
\hline De 64 y más & 7 & 12,7 \\
\hline $\begin{array}{l}\text { Total de } \\
\text { víctimas }\end{array}$ & 55 & 100 \\
\hline
\end{tabular}

Fuente: Datos obtenidos en la página web del Ministerio de sanidad, Política Social e Igualdad. Víctimas mortales por violencia de género. Ficha resumen- Datos provisionales. Fecha de actualización 28/11/2011. ${ }^{(7)}$

En la tabla III se exponen los datos referidos a las víctimas mortales por violencia de género según existencia de denuncia previa y/o medidas de protección durante el año 2011. Mientras en la tabla IV se muestra el mismo tipo de causa de muerte en relación con el tipo de pareja que tenían las mujeres durante el mismo periodo de tiempo.

Tabla III. Víctimas mortales por violencia de género según existencia de denuncia previa y/o medidas de protección. 2011

\begin{tabular}{|l|ll|}
\cline { 2 - 3 } \multicolumn{1}{c|}{} & $\begin{array}{l}\text { Node } \\
\text { casos }\end{array}$ & Porcentaje \\
\hline Habían denunciado & 14 & 25,5 \\
\hline Retiraron la denuncia & 1 & 1,8 \\
\hline $\begin{array}{l}\text { Solicitaron medidas de protección } \\
\text { Obtuvieron medida de protección }\end{array}$ & 9 & 16,4 \\
\hline $\begin{array}{l}\text { Renunciaron a medidas de } \\
\text { protección }\end{array}$ & 0 & 16,4 \\
\hline $\begin{array}{l}\text { Medidas de protección caducadas } \\
\text { Tenían medida de protección en } \\
\text { vigor }\end{array}$ & 7 & 0 \\
\hline Total víctimas & 55 & 1,8 \\
\hline
\end{tabular}


Fuente: Ministerio de sanidad, Política Social e Igualdad. Víctimas mortales por violencia de género. Ficha resumen-Datos provisionales. Fecha de actualización 28/11/2011. ${ }^{(8)}$

Tabla IV. Víctimas mortales por violencia de género según relación con la pareja. 2011.

\begin{tabular}{|l|ll|}
\cline { 2 - 3 } \multicolumn{1}{c|}{} & $\begin{array}{l}\text { № de Porcentaje } \\
\text { casos }\end{array}$ \\
\hline $\begin{array}{l}\text { Expareja o en fase de } \\
\text { ruptura }\end{array}$ & 23 & 41,8 \\
\hline Pareja & 32 & 58,2 \\
\hline Total víctimas & 55 & 100 \\
\hline
\end{tabular}

Fuente: Ministerio de sanidad, Política Social e Igualdad. Víctimas mortales por violencia de género. Ficha resumen-Datos provisionales. Fecha de actualización 28/11/2011. ${ }^{(9)}$

La gravedad de la situación en el panorama nacional motivó la realización de este estudio que pretende acercar el fenómeno de la violencia en la pareja, y en concreto al de malos tratos dirigidos a la mujer (porque esta es la víctima de esa violencia en un 95\% de los casos) ${ }^{(10)}$ a los distintos profesionales que en algún momento, por el cargo que ocupan, deban de enfrentar alguna situación de este tipo desde una perspectiva diferente: exponiendo los mecanismo de protección que el Estado proporciona a la sociedad para hacer frente a esta situación ya que para continuar con esta lucha es necesario conocer las armas que tenemos.

Es esencial que, tanto hombres como mujeres, y de manera especial los profesionales de Enfermería de nuestros servicios sanitarios, comprendan que la violencia dirigida a la mujer dificulta sobremanera el desarrollo de la sociedad y la consecución de objetivos referentes a su salud.

\section{MATERIAL Y MÉTODOS}

Para satisfacer el objetivo de nuestro estudio intentaremos exponer de manera resumida los distintos recursos con que el Estado dota a la sociedad para hacer frente a este tipo de maltrato. Para ello, hemos empleado (tras un proceso riguroso de selección) una ingente masa de bibliografía que nos ha valido de soporte para redactar esta revisión. Por ello, la técnica empleada es la de análisis de contenido, en la modalidad temática, que se basa en la lectura como instrumento de recogida de datos; lectura que, a diferencia de la lectura convencional, debe realizarse siguiendo el método científico, es decir, debe ser sistemática, objetiva, replicable y válida.

Para la confección de este estudio se adoptó como criterio de inclusión todos aquellos artículos y documentos de fuentes comprobadas y fiables que versan sobre la violencia contra la mujer. No hemos seleccionado un intervalo de tiempo concreto en el que basar la búsqueda de las distintas publicaciones pues dada la importancia histórica de algunos documentos nos ha sido por completo imposible aceptar este parámetro como un criterio de selección de información. A pesar de ello, en la elección de artículos de revistas sí que hemos sido menos condescendientes, pues hemos desechado todos aquellos cuya publicación haya sido anterior a la década de los 90 .

Se recurrió a varias fuentes de información entre las que destacamos: bases de datos con contenidos importantes para Enfermería como son SciELO, PubMed, Elsevier, Encuentr@ y Cochrane. Como descriptores de búsqueda en estas bases de datos se emplearon los términos: Violencia contra la mujer, violencia de género, igualdad de género y protección de 
la mujer maltratada. Asimismo se ha consultado el contenido de monografías de temática relacionada con el asunto abordado y que referenciamos en la bibliografía y de páginas electrónicas en internet de marcado interés para la elaboración del presente trabajo entre las que destacamos Nursing Network on Violence Against Women (NNVAWI), Ministerio de Sanidad, Política Social e Igualdad, Organización Mundial de la Salud (OMS), Boletín Oficial del Estado (BOE) y Sociedad Española de Medicina de Familia (semFYC).

\section{RESULTADOS Y DISCUSIÓN}

\section{Contexto histórico y legislativo de la violencia contra la mujer.}

Antes de comenzar a exponer los recursos mencionados con anterioridad hemos percibido la necesidad de exponer algunos de los eventos relacionados con la violencia de género más relevantes acontecidos en estas últimas cinco décadas junto a las aportaciones derivadas de ellos:

- La 49a Asamblea General celebrada en Ginebra en 1996 donde la OMS reconoció la violencia como un grave problema de salud pública, identificándola como factor crucial en el deterioro de la salud. ${ }^{(11,12)}$

- Conferencia Mundial de Derechos Humanos de Viena celebrada del 14 al 25 de junio de 1993 en la que se reconoce los Derechos de las mujeres como Derechos Humanos.

- Recomendación General número $19^{(14)}$ de la Convención sobre la eliminación de todas las formas de discriminación contra la mujer (CEDAW) ${ }^{(15)}$, aprobada en 1992 hace alusión sobre la violencia que afirma que "La Violencia contra la mujer es una forma de discriminación que impide gravemente el goce de derechos y libertades en pie de igualdad con el hombre".

Dos años más tarde, en 1995, tuvo lugar la IV Conferencia Mundial sobre la Condición Jurídica y Social de la Mujer, celebrada en Beijing, que inició un nuevo capítulo en la lucha por la igualdad entre los sexos al suponer el traslado del foco de atención de las mujeres al concepto de género, reconociendo que toda la estructura de la sociedad, y todas las relaciones entre los hombres y las mujeres en el interior de esa estructura, tenían que ser reevaluadas. $^{(16)}$

- De la anterior conferencia surgió la Plataforma de Acción de Beijing, formada por 189 representantes de gobierno. En ella se identificaron doce esferas de especial preocupación que se estimaba que representaban los principales obstáculos al adelanto de la mujer y que exigían la adopción de medidas concretas por parte de los gobiernos y se menciona las medidas concretas que deben de adoptar los distintos gobiernos, la comunidad internacional, las organizaciones no gubernamentales y el sector privado para sortear dichos obstáculos, entre esas doce esferas se encuentra la violencia contra las mujeres. ${ }^{(17)}$ Así, ya desde 1995, en el seno de Naciones Unidas se reconoce que la violencia de género se constituye como uno de los principales obstáculos para el abordaje de la libertad, el desarrollo y el disfrute de los derechos de la Mujer. ${ }^{(18)}$

El lugar de las mujeres en la sociedad se puede analizar a través de las leyes y, en este sentido, la evolución de la legislación española sobre la violencia de género aporta datos significativos. 
La transición a la democracia cambia todo el marco jurídico español, en el que la Constitución del año 1978 reconoce expresamente la igualdad legal entre hombres y mujeres. La dignidad de la persona [art. 14, Constitución Española] ${ }^{(19)}$, el derecho a la vida y a la integridad física y moral [art. 15, Constitución Española] ${ }^{(20)}$, el derecho del hombre y la mujer a contraer matrimonio con plena igualdad jurídica [art. 32, Constitución Española] ${ }^{(21)}$, la protección social, económica y jurídica de la familia, de los hijos, con independencia de su filiación, y de las madres [art. 39, Constitución Española] se erigen en valores básicos de la convivencia. Y, paulatinamente, estos nuevos principios de la doctrina constitucional se van introduciendo mediante sucesivas reformas en todos los textos legales.

La Ley 30/1981 de 7 de julio por la que se modifica la regulación del matrimonio en el Código Civil y se determina el procedimiento a seguir en las causas de nulidad, separación y divorcio tuvo una relevancia fundamental ya que supuso la posibilidad de poder romper la relación establecida entre el hombre y la mujer cuando dicha relación es destructiva. ${ }^{(22)}$

En relación con la legislación existente en relación con la violencia de género encontramos a nivel estatal leyes como la Ley de Enjuiciamiento Criminal, debiendo destacarse la Ley Orgánica 11/2003, de 29 de septiembre de Medidas Concretas en Materia de Seguridad Ciudadana, Violencia Doméstica e Integración Social de Extranjeros, ${ }^{(23)}$ así como la Ley Orgánica 15/2003, de 25 de noviembre, por la que se modifica la Ley Orgánica 10/1995, de 23 de noviembre, del Código Penal. ${ }^{(24)}$ Las herramientas jurídicas de suma importancia, al permitir la protección integral e inmediata de las víctimas de malos tratos, son la Ley 27/2003, de 31 de julio, Reguladora de la Orden de Protección de las víctimas de la violencia de género ${ }^{(25)}$ y la Ley Integral contra la violencia de género ${ }^{(26)}$ que pretende que la sociedad española sea activa con un cambio de actitud que rechace cualquier forma de malos tratos dentro de la relación de pareja. Esta ley abarca aspectos preventivos, educativos, sociales, asistenciales, sanitarios y penales. En la ley se articulan una serie de medidas legislativas cuyo propósito es alcanzar una serie de objetivos, tales como: el fortalecimiento de la sensibilidad ciudadana ante la violencia de género; el reconocimiento de los derechos subjetivos de las víctimas de la violencia, el establecimiento de un sistema de servicios sociales de atención y de recuperación de las víctimas; la garantía de una serie de derechos económicos y de derechos laborales a las víctimas de la violencia de género; el establecimiento de un sistema institucional de tutela; fortalecimiento del marco penal y procesal, así como la coordinación de todos los recursos dedicados a este problema social.

Tomando como referencia las reflexiones internacionales en torno a la violencia de género, España aprueba, por unanimidad de todos los grupos parlamentarios, la Ley Orgánica 1/2004, de 28 de diciembre, de Medidas de Protección Integral contra la Violencia de Género. ${ }^{(27)}$ Esta Ley tiene por objeto:

- Actuar contra la violencia que los hombres ejercen sobre las mujeres (bien sean o hayan sido cónyuges, bien hayan estado ligados a ellas por relaciones similares de afectividad, aun sin convivencia).

- Establecer medidas de protección integral cuya finalidad es prevenir, sancionar y erradicar la violencia contra la mujer de cualquier tipo y prestar asistencia a sus víctimas.

\section{¿Qué recursos suministra el Estado?}

Es importante que los profesionales sanitarios conozcan estos recursos ya que en determinadas ocasiones la mujer puede estar desinformada al respecto por no ser suficientes las notificaciones por otros medios. Además es fundamental para que sean 
capaces de confeccionar una pauta de actuación pertinente, pues muchas veces estas víctimas hacen un uso más reiterado de los servicios sanitarios ${ }^{(28,29,30,31)}$ y se crea una oportunidad excepcional en las consultas de atención, tanto primaria como especializada, de iniciar una relación terapéutica en la que el suministro de información a la mujer por parte del profesional se hace necesaria. Algunos de estos recursos son:

Guía de derechos: en esta guía se expone una definición de violencia de género y exponen de forma completa los derechos que la mujer posee para que ella, una vez los conozca, pueda ejercerlos de forma efectiva. Está publicada en varios idiomas.

La Web de recursos de apoyo y prevención ante casos de violencia de género (WRAP) ${ }^{(32)}$ constituye un servicio integrado en la página Web del Ministerio de Sanidad, Política Social e Igualdad que permite la localización sobre mapas activos de los distintos recursos (policiales, judiciales y de información, atención y asesoramiento) que las administraciones públicas y las entidades sociales han puesto a disposición de la ciudadanía y de las víctimas de violencia de género.

Llamadas al 016 por violencia de género. Servicio telefónico de información y asesoramiento jurídico en materia de violencia de género: servicio disponible las 24 horas del día los 365 días del año, aplicado en todo el territorio español, atendido por personas con conocimientos demostrados en el tema y adaptado a la situación personal de las víctimas de violencia de género que garantiza el cumplimiento del derecho de información reconocido en el artículo 18 de la Ley Integral a las mujeres víctimas de violencia de género garantizando en cualquier caso la confidencialidad de los datos prestados y derivando automáticamente aquellas llamadas de emergencia y/o urgencia a los centros 112 autonómicos. La prestación de este servicio se realiza en 51 idiomas diferentes.

Usuarias del servicio de teleasistencia móvil para víctimas de violencia de género: El Instituto de Mayores y Servicios Sociales (IMSERSO) y la Federación Española de Municipios y Provincias (FEMP) suscribieron con fecha 4 de octubre de 2004 una Addenda, dentro del Convenio Marco suscrito entre ambas instituciones el 20 de abril de 1993, para la implantación de un servicio de teleasistencia domiciliaria para personas mayores y discapacitadas, para la implantación, a cargo del IMSERSO, del Servicio de Teleasistencia Móvil a las víctimas de violencia de género que cuenten con medidas judiciales de protección. Este servicio comenzó a funcionar en diciembre de 2005 y las empresas adjudicatarias del mismo fueron Cruz Roja Española y Eulen Servicios Sociosanitarios. Consiste en un servicio telefónico de información permanente que ofrece a las víctimas de la violencia de género una atención inmediata y a distancia, asegurando una respuesta rápida a las eventualidades que puedan sobrevenir las 24 horas del día. A la vez proporciona tranquilidad y seguridad, potencia la autoestima y la calidad de vida de las usuarias del servicio.

Este servicio puede ser solicitado por mujeres víctimas de violencia de género con orden de alejamiento o de protección (siempre que el juez que la emitió no la crea contraproducente) que no conviva con la persona o personas que las han sometido o maltratado. Puede ser solicitada en cualquier momento del año y es de carácter gratuito. La documentación pertinente se entregará en el ayuntamiento de residencia.

Contratos bonificados de mujeres víctimas de violencia y contratos de sustitución de víctimas de violencia de género: La Ley Orgánica 1/2004, de 28 de diciembre, consagra y garantiza a las mujeres víctimas de violencia de género, una serie de derechos laborales y 
de Seguridad Social, con la finalidad de conciliar sus obligaciones laborales con sus necesidades de protección y de recuperación integral. ${ }^{(33)}$

Asimismo, el Real Decreto 1917/2008, de 21 de noviembre, por el que se aprueba el programa de inserción sociolaboral para mujeres víctimas de violencia de género, contempla medidas activas de empleo al objeto de dotar a las víctimas de mayores facilidades para acceder al empleo, incrementar su autonomía personal y poner a su disposición todos los recursos creados al efecto. ${ }^{(34)}$

Percepción de la renta activa para la inserción (RAl): El programa de la Renta Activa de Inserción (RAI), forma parte de la acción protectora por desempleo del régimen público de Seguridad Social y, de acuerdo con las directrices sobre el empleo de la Unión Europea, comprende no solamente el pago de una prestación, sino también acciones específicas de formación, perfeccionamiento, orientación, reconversión e inserción profesional. ${ }^{(35)}$

Tiene por objeto incrementar las oportunidades de retorno al mercado de trabajo de los trabajadores desempleados con especiales necesidades económicas y dificultades para encontrar empleo. Las mujeres víctimas de violencia de género pueden solicitarla si cumplen con unas obligaciones como aceptar la colocación adecuada que les sea ofrecida, renovar la demanda de empleo según se determine, buscar activamente empleo, etc.

La cuantía de la renta activa de inserción es igual al 80\% del indicador público de renta de efectos múltiples (IPREM) mensual vigente en cada momento y se percibirá como máximo durante 11 meses. Es incompatible con la ayuda económica prevista en el artículo 27 de la Ley Integral que se expone a continuación.

Ayuda económica prevista en el artículo 27 de la Ley Integral: El artículo 27 de la Ley $1 / 2004$, desarrollado por el Real Decreto $1452 / 2005,{ }^{(36)}$ garantiza a las víctimas de violencia de género la percepción de una ayuda social, cuando se sitúen en un determinado nivel de rentas y se presuma que, debido a su edad, falta de preparación general o especializada y circunstancias sociales, la víctima tendrá especiales dificultades para obtener un empleo y siempre que carezca de rentas que, en cómputo mensual, superen el 75 por ciento del salario mínimo interprofesional vigente, excluida la parte proporcional de dos pagas extraordinarias o que presente especiales dificultades para obtener un empleo, que se acreditará a través del informe del Servicio Público de Empleo. El importe de esta ayuda es, con carácter general, equivalente al de seis meses de subsidio por desempleo. No obstante, esta ayuda puede tener distintas cuantías, de doce, dieciocho o veinticuatro meses del importe de dicho subsidio, en función de las responsabilidades familiares de la beneficiaria y, en su caso, del grado de minusvalía que tenga oficialmente reconocida, igual o superior al $33 \%$ (tanto la víctima como los familiares a su cargo o menores acogidos con los que conviva).

El objeto de este recurso es facilitar la integración social y autonomía personal.

Son causas de reintegro de la ayuda los supuestos en los que la solicitante la hubiera obtenido sin reunir los requisitos exigidos para su concesión, o falseando u ocultando los hechos o fatos que hubieran podido ser la causa de su denegación.

Sistema de seguimiento por medios telemáticos de las medidas de alejamiento en el ámbito de la violencia de género: El artículo 64.3 de la Ley Orgánica 1/2004, de 28 de diciembre, de Medidas de Protección Integral contra la Violencia de Género, ${ }^{(37)}$ contempla, entre las medidas cautelares y de aseguramiento, la consistente en prohibir al inculpado que 
se aproxime a la persona protegida y prevé, además, que podrá acordarse la utilización de instrumentos de tecnología adecuada para verificar de inmediato el incumplimiento de esta Medida de alejamiento.

La implantación de los dispositivos electrónicos se ha hecho efectiva el 24 de julio de 2009 en todo el territorio del Estado español.

Servicio telefónico (900 $2100 \quad 21$ ) y on-line de información y orientación sobre políticas de igualdad y hombres: Este servicio tiene como objetivo general informar sobre todas las cuestiones que puedan interesar a los hombres en relación con las políticas de igualdad, y se puso en funcionamiento el 4 de diciembre de 2009. Tiene un doble objetivo:

- Contribuir a avanzar en la consecución de relaciones más igualitarias entre mujeres y hombres.

- Aunar esfuerzos en la lucha contra la violencia de género, máxima expresión de la desigualdad entre hombres y mujeres.

Este servicio atiende todas las llamadas desde cualquier punto de España y se presta en castellano, si bien existe una franja de 4 horas, una tarde a la semana, para atender las llamadas en el resto de lenguas cooficiales. El servicio es gratuito y el horario de atención es de lunes a viernes, de 9 a 23 horas. Por su parte, las consultas on-line se deberán contestar en un plazo máximo de 72 horas desde su recepción.

\section{CONCLUSIONES}

Con este estudio esperamos haber puesto de manifiesto las siguientes conclusiones:

Una relación de pareja está orientada para compartir y disfrutar de la vida con la persona que se enamora de nosotros y de la cual nosotros nos enamoramos. No está legitimada la exclusión de uno de los miembros de algún derecho al igual que tampoco se aprueba la capacidad de una de las partes para subyugar la autonomía de la otra parte: la violencia contra la mujer es un proceder que se encuentra no solo socialmente, sino también legalmente rebatido.

- La Violencia de Género constituye en cualquiera de los casos una vulneración de los derechos fundamentales de la persona, derechos que son recogidos en las distintas fuentes de carácter legislativo consultadas. Es necesario actuar contra ella: La legislación y la educación juega un papel fundamental en este punto.

La legislación y las diversas medidas de protección adoptadas a raíz de ella para hacer frente a la violencia de género son el resultado de un intento de concienciar a la sociedad y de una lucha anterior por los derechos que la mujer posee; reivindicaciones que se muestran a lo largo del bagaje histórico en los numerosos eventos y documentos, algunos de los cuales hemos expuesto de manera resumida en el presente trabajo.

Cuando se desvela un caso de violencia contra la mujer en la consulta de atención sanitaria se presenta una oportunidad única para la enfermera de crear una relación terapéutica con la que la mujer agredida se sienta segura. Este es uno de los motivos más importantes por los que el profesional sanitario debe de tener una preparación y unos conocimientos actualizados en materia de legislación, de perspectiva histórica y de recursos 
aplicables en materia de salud y género para hacer frente de la manera más eficiente posible al problema.

Todos los recursos aportados por el Estado mencionados son relevantes pero no debemos de olvidar uno en absoluto desdeñable: el equipo interdisciplinar sanitario entre el que se incluye el conjunto de Enfermería y que, siendo conocedor de estos recursos, puede convertirse en un aliado destacado a la hora de garantizar la seguridad e integridad de la mujer.

De la anterior conclusión se deduce que la formación de los profesionales enfermeros en el conocimiento de las medidas de protección instauradas así como el trabajo en equipo son fundamentales para hacer frente a este problema que repercute negativamente en la salud de la sociedad.

A la profesión enfermera le queda un largo camino por recorrer para comprender este fenómeno tan complejo. Es importante reconocer su labor en la identificación de los casos, la elaboración de un plan de cuidados integrado e individualizado y de la educación de la sociedad.

\section{REFERENCIAS}

1. Ministerio de Sanidad, Política Social e Igualdad. Violencia de género. ¿Qué es? [Internet]. S.f. [Citado 2011 Jun 15]; Disponible en: http://www.seigualdad.gob.es/violenciaGenero/queEs/home.htm

2. Palomo Pinto, M. Violencia contra la mujer: atención urgente. Ante el maltrato ¿debe prevalecer la seguridad de la mujer o la legalidad vigente? Rev. Semergen [revista en la internet]. 2003 [citado 2011 Jun 15]; 30 (2): 68-71. Disponible en: http://www.elsevier.es/es/revistas/semergen-medicina-familia-40/violencia-mujer-atencionurgente-ante-maltrato-debe-13057632-articulo-opinion-2004

3. Vives, C; Álvarez-Dardet, C; Caballero, P. Violencia del compañero íntimo en España. Rev. Gac Sanit. [Revista en la internet]. 2003; 17 (4): 268-74. Disponible en: http://www.elsevier.es/es/revistas/gaceta-sanitaria-138/violencia-compa\%C3\%B1ero-intimoespa\%C3\%B1a-13051043-originales-2003

4. INE. Víctimas mortales por violencia de género. [Internet] 2011 [Citado 2011 nov 27]. Disponible

en: http://www.ine.es/jaxi/tabla.do?type=pcaxis\&path=/t00/mujeres hombres/tablas 1/l0/\&file=v0 7002.px

5. Ministerio de Igualdad. Víctimas mortales por violencia de género ficha resumendatos provisionales. [Internet]. 2011 [Citado 2011 nov 29]. Disponible en: http://www.migualdad.es/ss/Satellite?blobcol=urldata\&blobheader=application\%2Fpdf\&blobh eadername $1=$ Content-

disposition\&blobheadervalue $1=$ inline\&blobkey=id\&blobtable=MungoBlobs\&blobwhere $=12446$ $\underline{55769613 \& \text { ssbinary=true }}$

6. INE. Loc. Cit. Ver referencia 4.

7. Ministerio de Igualdad. Loc. Cit. Ver referencia 5.

8. Ibid.

9. $\quad$ Ministerio de Igualdad. Loc. Cit. Ver referencia 5.

10. López Mondéjar Lola. Una patología del vínculo amoroso: el maltrato a la mujer. Rev. Asoc. Esp. Neuropsiq. [revista en la Internet]. 2001 Mar [citado 2011 Jun 15]; (77): 7-26. Disponible en: http://scielo.isciii.es/scielo.php?script=sci_arttext\&pid=S021157352001000100002\&Ing=es. doi: 10.4321/S0211-57352001000100002. 
11. Blanco, Pilar; Ruiz-Jarabo, Consuelo; García de Vinuesa, Leonor; Martín-García, Mar. La violencia de pareja y la salud de las mujeres, Rev. Gac. Sanit. [revista en la internet]. 2004 [citado 2011 Jun 15]; 18 (Supl 1): 182-8. Disponible en: http://www.elsevier.es/es/revistas/gacetasanitaria-138/la-violencia-pareja-salud-las-mujeres-13062524-capitulo-1-visiones-saludpublica-perspectiva-genero-clase-social-2004

12. World Health Organization. World Report on violence Health, Ginebra. [Internet]. 2002 [citado 2011 Jun 15]; Disponible http://www5.who.int/violence injury prevention/download.cfm?id=0000000582.

13. Naciones Unidas. Declaración y programas de acción de Viena. Procedente de la Conferencia Mundial de Derechos Humanos; 1993; jun 14-25; Viena (Austria); [Internet] 1993 [citado 2011 Jun 15]; Disponible http://www.unhchr.ch/huridocda/huridoca.nsf/(Symbol)/A.CONF.157.23.Sp

14. CEDAW. Recomendación general 19, adoptada por el Comité para la Eliminación de la Discriminación contra la Mujer, $11^{\circ}$ período de sesiones, 1992, U.N. [Internet] 1992 [citado 2011 Jun 15]; Disponible en: http://www1.umn.edu/humanrts/gencomm/Sgeneral19.htm

15. Naciones Unidas. Convención sobre la eliminación de todas las formas de discriminación contra la mujer; 1979. S.I. [Internet] 1979 [citado 2011 Jun 15]; Disponible en: http://www2.ohchr.org/spanish/law/cedaw.htm

16. Naciones Unidas. Informe de la Cuarta Conferencia Mundial sobre la Mujer; 1995 sept 4-15; Beijing (China); [Internet] 1995 [Citado 2011 Jun 16]; Disponible en: http://www.un.org/womenwatch/daw/beiijing/pdf/Beiijing\%20full\%20report\%20S.pdf

17. Naciones Unidas. Periodo Extraordinario de Sesiones de la Asamblea General de las Naciones Unidas: Igualdad entre los géneros, desarrollo y paz en el siglo XXI. 2000 jun 5-9; Nueva York (USA). [Interent] 2000 [Citado 2011 jun 18]; Disponible en: http://www.un.org/spanish/conferences/Beijing/mujer2021.htm

18. Ministerio de Sanidad, Política Social e lgualdad. Violencia de género ¿Qué es? [Internet] S.f. [Citado 2011 jun 21]; Disponible en: http://www.inmujer.es/ss/Satellite?cid=1193047406897\&pagename=Ministeriolgualdad\%2FP age\%2FMIGU contenidoFinal

19. Anón. Capítulo II Derechos y libertades: artículo 14. En: Constitución Española. [Internet]. 1aㅡ edición. España; Editorial MAD; 1978 [citado 2011 jun 25]. Disponible en: http://books.google.es/books?id=hxwohRUlfbkC\&printsec=frontcover\&dq=constituci\%C3\%B3 n+espa\%C3\%B1ola\&hl=es\&sa=X\&ei=y3ouT92pFM-

XhQecmunLCg\&ved=0CD0Q6AEwAQ\#v=onepage\& $q=$ constituci\%C3\%B3n\%20espa\%C3\%B 1ola\&f=false

20. Anón. Capítulo II Derechos y libertades: artículo 15. En: Constitución Española. [Internet]. 1a edición. España; Editorial MAD; 1978 [citado 2011 jun 25]. Disponible en: http://books.google.es/books?id=hxwohRUlfbkC\&printsec=frontcover\&dq=constituci\%C3\%B3 n+espa\%C3\%B1ola\&hl=es\&sa =X\&ei=y3ouT92pFM-

XhQecmunLCg\&ved=0CD0Q6AEwAQ\#v=onepage\&q=constituci\%C3\%B3n\%20espa\%C3\%B 1ola\&f=false

21. Anón. Capítulo II Derechos y libertades: artículo 32. En: Constitución Española. [Internet]. 1aㅡ edición. España: Editorial MAD; 1978 [citado 2011 jun 25]. Disponible en: http://books.google.es/books?id=hxwohRUlfbkC\&printsec=frontcover\&dq=constituci\%C3\%B3 n+espa\%C3\%B1ola\&hl=es\&sa=X\&ei=y3ouT92pFM-

XhQecmunLCg\&ved=0CD0Q6AEwAQ\#v=onepage\& $\mathrm{q}=$ constituci\%C3\%B3n\%20espa\%C3\%B 1ola\&f=false

22. Anón. Ley 30/1981 de 7 de julio por la que se modifica la regulación del matrimonio en el Código Civil y se determina el procedimiento a seguir en las causas de nulidad, separación y divorcio. España: BOE; [Internet] 1981 [Citado 2011 jun 26]. Disponible en: http://www.boe.es/boe/dias/1981/07/20/pdfs/A16457-16462.pdf 
23. Anón. Ley Orgánica 11/2003, de 29 de septiembre de medidas concretas en materia de seguridad ciudadana, violencia doméstica e integración. España: BOE; [Internet] 2003 [Citado 2011 jun 26]. Disponible en: http://www.boe.es/boe/dias/2003/09/30/pdfs/A3539835404.pdf

24. Anón. Ley Orgánica $15 / 2003$, de 25 de noviembre por la que se modifica la Ley Orgánica 10/1995, de 23 de noviembre del Código Penal. España: BOE; [Internet] 2003 [Citado 2011 jun 26]. Disponible en: http://www.boe.es/boe/dias/2003/11/26/pdfs/A4184241875.pdf

25. Anón. Ley 27/2003, de 31 de julio, reguladora de la Orden de protección de las víctimas de la violencia doméstica. España: BOE; [Internet] 2003 [Citado 2011 jun 26]. Disponible en: http://www.boe.es/boe/dias/2003/08/01/pdfs/A29881-29883.pdf

26. Anón. Ley $5 / 2005$ de 20 , de diciembre, Integral contra la violencia de género de la Comunidad de Madrid. España: BOE; [Internet] 2006 [Citado 2011 jun 26]. Disponible en: http://www.boe.es/boe/dias/2006/03/02/pdfs/A08515-08526.pdf

27. Anón. Ley Orgánica 1/2004, de 28 de diciembre, de Medidas de Protección Integral contra la Violencia de Género. España: BOE; [Internet] 2004 [Citado 2011 jun 26]. Disponible en: http://www.boe.es/boe/dias/2004/12/29/pdfs/A42166-42197.pdf

28. Blanco P. Op. Cit. p. 20. Ver referencia 11.

29. Campbell JC. Health consequences of intimate partner violence. Lancet [Internet]. 2002 [citado 2011 Jun 15];359:1331-6. Disponible en: http://www.nnvawi.org/pdfs/alo/campbell 1.pdf

30. Rohlfs Izabella, Valls-Liobet. Actuar contra la violencia de género: un reto para la salud pública. Rev. Gac. Sanit. [revista en la internet]. 2003 [citado 2011 Jun 15]; 17 (4): 263-5. Disponible en: http://www.elsevier.es/es/revistas/gaceta-sanitaria-138/actuar-violencia-genero-un-retosalud-publica-13051041-editoriales-2003

31. Ruiz-Pérez, Isabel; Plazaola-Castaño, Juncal; Blanco-Prieto, Pilar; GonzálezBarranco, Juana María;Ayuso-Martín, Pilar; Montero-Piñar, María Isabel. La violencia contra la mujer en la pareja. Un estudio en el ámbito de la atención primaria. Rev. Gac. Sanit. [revista en la internet]. 2006 [citado 2011 Jun 15]; 20 (3): 202-8. Disponible en: http://www.elsevier.es/es/revistas/gaceta-sanitaria-138/la-violencia-mujer-pareja-un-estudioambito-13088851-originales-2006

32. http://www.migualdad.es/recursos/search/SearchForm.action. [Accesed June 26, 2011].

33. Anón. Loc. Cit. Ver referencia 27.

34. Anón. Real Decreto 1917/2008, de 21 de noviembre, por el que se aprueba el programa de inserción sociolaboral para mujeres víctimas de violencia de género. España: BOE; [Internet] 2008 [Citado 2011 jun 26]. Disponible en: http://www.boe.es/boe/dias/2008/12/10/pdfs/A49367-49373.pdf

35. Anón. Real Decreto 205/2005, de 25 de febrero, por el que se regula para el año 2005 el programa de renta activa de inserción para desempleados con especiales necesidades económicas y dificultades para encontrar empleo. España: BOE; [Internet] 2005 [Citado 2011 jun 26]. Disponible en: http://www.boe.es/boe/dias/2005/02/26/pdfs/A07086-07091.pdf

36. Anón. Op. Cit. p. 22. Ver referencia 27.

37. Ibid.

\section{BIBLIOGRAFÍA CONSULTADA}

1. Alonso A. Atención Primaria y Salud Mental. 2000:3;4:4-9.

2. Arrufat A, Criado J, Colomer C, Albert S, Sánchez R. Denuncias por agresión a mujeres en la Plana Baixa (Castellón). Gac Sanit 2003;17:343. 
3. Artazcoz, Lucía. Las desigualdades de género en salud en la agenda de salud pública. Rev. Gac. Sanit. [revista en la internet]. 2004 [citado 2011 Jun 15]; 18(Supl 2): 1-2. Disponible en: http://www.elsevier.es/es/revistas/gaceta-sanitaria-138/las-desigualdades-genero-saludagenda-salud-publica-13061988-editoriales-2004

4. $\quad$ Blanco Prieto, P; Ruíz Jarabo, C; La prevención y detección de la violencia contra las mujeres en la atención primaria de salud. Madrid: Editorial para la asociación y la defensa de la sanidad pública de Madrid. 2002.

5. Bordieu, P: La dominación masculina. Barcelona: Anagrama. 2000.

6. Bonino, L.; Varones, género y salud mental, ponencia presentada en las $X$ Joranadas de la Asociación Española de Neuropsiquiatría, Palma, noviembre de 1999.

7. Campbell JC. Health consequences of intimate partner violence. Lancet [Internet]. 2002 [citado 2011 Jun 15];359:1331-6. Disponible en: http://www.nnvawi.org/pdfs/alo/campbell 1.pdf

8. Cano Martín Luis Miguel, Berrocoso Martínez Alberto, Arriba Muñoz Leonor de, Bernaldo de Quirós Lorenzana Rodrigo, Alamar Provecho Juan Diego, Cardo Maza Ana. Prevalencia de Violencia de Género en mujeres que acuden a consultas de Atención Primaria. Rev Clin Med Fam [revista en la Internet]. 2010 Feb [citado 2011 Jun 15] ; 3(1): 10-17. Disponible en: http://scielo.isciii.es/scielo.php?script=sci_arttext\&pid=S1699695X2010000100004\&lng=es. doi: 10.4321/S1699-695X2010000100004.

9. Carotozzolo, D.; La pareja violenta. Una lectura psicoanalítica. Rosario: Homo Sapiens. 1997.

10. Cayuela Soler Alba, Gimeno Cardells. Violencia de género, una enfermedad infecciosa. Revista Educare. [revista en internet]. 2007 [citado 2011 Jun 15]; 5 (39): 61-67. Disponible

http://encuentra.enfermeria21.com/component/encuentra/?task=showContent main\&q=pclav e\%5Bviolencia+de+genero\%5D\&search type=10\&search entity=pclave\&id pub grp=0\&id pub cont $=4 \&$ id articulo $=39032$

11. Cobo Jesús, Muñoz Ruth, Martos Ascensión, Carmona Monserrat, Pérez Mireia, Cieici Roser, García- Parés Gemma. La violencia de género en la atención psiquiátrica y psicológica especializada: ¿es relevante la violencia de género para nuestros profesionales? Revista de psiquiatría y salud mental [revista en la internet]. 2010 [citado 2011 Jun 15]; 3 (2): 61-67. Disponible en: http://www.elsevier.es/es/revistas/revista-psiquiatria-salud-mental-286/la-violencia-genero-atencion-psiquiatrica-psicologica-especializada-13151590-originalbreve-2010

12. Coll- Vinent Blanca, Echevarria Teresa, Rodríguez Dolores, Ferrás Ursula, Millá José, Santiñá Manuel. Percepción de la violencia de género por el personal sanitario. Rev. Gac. Sanit. [revista en la internet]. 2008 [citado 2011 Jun 15]; 22(6): 618-20. Disponible en: http://www.elsevier.es/es/revistas/gaceta-sanitaria-138/percepcion-violencia-genero-

personal-sanitario-13129690-cartas-al-director-2008.

13. Corsi J. Violencia familiar, una mirada interdisciplinar sobre un grave problema social. Buenos Aires: Ed. Paidós, 1994.

14. Corsi J. Violencia masculina en la pareja. Buenos Aires: Ed. Paidós, 1995.

15. Echeburia, E.; De Corral, P.; Manual de violencia familiar. Madrid: Siglo XXI editores. 1998.

16. Echeburúa E., Fernández Montalvo J., Paz de Coral. "Predicción del riesgo de homicidios y de violencia grave en relación de pareja. Instrumentos de evaluación de riesgo y adopción de medidas de protección”. Centro Reina Sofía. 2009.

17. Escudero Nafs Antonio, Polo Usaola Cristina, López Gironés Marisa, Aguilar Redo Lola. La persuasión coercitiva, modelo explicativo del mantenimiento de las mujeres en una situación de violencia de género: I: Las estrategias de la violencia. Rev. Asoc. Esp. Neuropsiq. [revista en la Internet]. 2005 Sep [citado 2011 Jun 15] ; (95): 85-117. 
Disponible en: $\quad$ http://scielo.isciii.es/scielo.php?script=sci arttext\&pid=S021157352005000300006\&lng=es. doi: 10.4321/S0211-57352005000300006.

18. Fernández Alonso MC, Herrero Velásquez S, Buitrago Ramírez F, Ciurana Misol R, Chocrón Bentata L, García Campayo J, et al. "Violencia Doméstica". Madrid: SEMFYC; 2003.

19. Fernández Alonso MC. Violencia doméstica: ¿conocemos los efectos de la violencia sobre la salud de las víctimas? Aten Primaria. 2004;34:125-7.

20. García Moreno C. Dilemes and opportunities for an apropiate health service response to violence against women. Lancet 2002;359:1509-14.

21. García Torrecillas JM, Torío Durántez J, lea Pereira MC, García Tirado MC, Aguilera Tejero R. Detección de violencia contra la mujer en la consulta del médico de familia. Aten Primaria. 2008; 40(9):455-61.

22. González Lozoya Inmaculada, Serrano Martínez Ana, García Sánchez Nuria, Campo Giménez María del, Moreno Ruiz Beatriz, González Lozoya Ana Belén et al . Experiencias en relación con la Violencia de Género de la población que consulta en Atención Primaria. Rev Clin Med Fam [revista en la Internet]. 2010 [citado 2011 Jun 15]; 3(2): 104-109. Disponible en: $\quad$ http://scielo.isciii.es/scielo.php?script=sci arttext\&pid=S1699695X2010000200009\&lng=es. doi: 10.4321/S1699-695X2010000200009.

23. Gunter, Jennifer. Violencia sexual en la pareja. Rev. Obstet. Gynecol. Clin. N. Am. 2007; 34 (3): 367-88.

24. Heritier, F.; Masculino/ Femenino. El pensamiento de la diferencia, Barcelona: Ariel. 1996.

25. Hernández Cordero E, Casla Villacorta M.E.Violencia de género: lesiones incisas múltiples, a propósito de un caso que se asemeja a la tortura. Med. Legal [revista en la $\begin{array}{lllllll}\text { internet]. } 2010 & \text { [citado } 2011 \text { Jun 15]; } 36 & \text { (3): 127-128. Disponible en: }\end{array}$ http://www.elsevier.es/es/revistas/revista-espa\%C3\%B1ola-medicina-legal-285/violenciagenero-lesiones-incisas-multiples-proposito-un-13189922-medicina-legal-imagenes-2010 26. Lorente Acosta M, Lorente Acosta J A. Agresión a la mujer: maltrato, violación y acoso. Granada: Ed. Comares, 1998.

27. Lorente Acosta Miguel. Medicina Legal y forense y violencia de género. Rev. Medicina legal [revista en la internet]. 2010 [citado 2011 Jun 15]; 36 (3): 91-92. Disponible en: http://www.elsevier.es/es/revistas/revista-espa\%C3\%B1ola-medicina-legal-285/medicinalegal-forense-violencia-genero-13189915-editorial-2010

28. Lorente Acosta, M. Mi marido me pega lo normal. 1eㅡ. Barcelona: Booket; 2009.

29. Melguizo, Miguel. Violencia de género. Hacer visible la vergüenza. Rev. Aten. Primaria. 2010; 42 (2): $77-78$.

30. Muñoz-Cobos, F; Burgos-Varo, ML; Carrasco-Rodríguez, A; Martín-Carretero, ML; Río-Ruiz, J;Villalobos-Bravo, M; Ortega-Fraile, I. Investigación cualitativa en mujeres víctimas de violencia de género. Rev Aten. Primaria. 2006; 37 (7): 407-12.

31. Plan de acción contra la violencia doméstica 1998-2000. 2ª reimpr Madrid: Instituto de la Mujer. Ministerio de Trabajo y Asuntos sociales, 2000.

32. Plazaola-Castaño J, Ruiz-Pérez I. Violencia contra la mujer en la pareja y consecuencias en la salud física y psíquica. Med Clin (Barc). 2004;122: 461-7.

33. Protocolo común para la actuación sanitaria ante la violencia de género. Comisión Contra la Violencia de Género del Consejo Interterritorial del Sistema Nacional de Salud. Ministerio de Sanidad y Consumo; [Internet] 2007 [citado 2011 Jun 15]; Disponible en: http://www.msc.es/organizacion/sns/planCalidadSNS/pdf/equidad/protocoloComun.

34. Raya Ortega L, Ruiz Pérez J, Plazaola Castaño J, Brun López-Abisab S, Rueda Lozano D, García de Vinuesa L, et al. La violencia contra la mujer en la pareja como factor asociado a una mala salud física y psíquica. Aten Primaria. 2004; 34:117-27.

35. Rhodes N, Baranoff McKenzie E,; Why do battered women stay? Three decades of research, Rev. Agrression and Violent Behavior. 1998; 3 (4): 107- 113. 
36. Rohlfs Izabella, Borrel C, Ponseca MC. Género desigualdades y salud pública, conocimientos y desconocimientos. Rev. Gac. San. 2000; 14 (Supl 3): 70-71.

37. Rohlfs Izabella, Valls-Liobet. Actuar contra la violencia de género: un reto para la salud pública. Rev. Gac. Sanit. [revista en la internet]. 2003 [citado 2011 Jun 15]; 17 (4): 263-5. Disponible en: http://www.elsevier.es/es/revistas/gaceta-sanitaria-138/actuar-violencia-genero-un-retosalud-publica-13051041-editoriales-2003

38. Romero Inmaculada. Intervención en Violencia de Género: Consideraciones en Torno al Tratamiento. Intervención Psicosocial [revista en la Internet]. 2010 Jul [citado 2011 Jun 15]; 19(2): 191-199. Disponible en: http://scielo.isciii.es/scielo.php?script=sci arttext\&pid=S1132-05592010000200010\&lng=es.

39. Ruiz-Pérez I, Blanco-Prieto P, Vives-Cases C. Violencia contra la mujer en la pareja: determinantes y respuestas sociosanitarias. Gac Sanit. 2004;18 Supl 2:4-12.

40. Ruiz-Pérez, Isabel; Plazaola-Castaño, Juncal; Blanco-Prieto, Pilar; GonzálezBarranco, Juana María;Ayuso-Martín, Pilar; Montero-Piñar, María Isabel. La violencia contra la mujer en la pareja. Un estudio en el ámbito de la atención primaria. Rev. Gac. Sanit. [revista en la internet]. 2006 [citado 2011 Jun 15]; $20 \quad$ (3): 202-8. Disponible en: http://www.elsevier.es/es/revistas/gaceta-sanitaria-138/la-violencia-mujer-pareja-un-estudioambito-13088851-originales-2006

41. Rodríguez Luengo R M. Abordaje enfermero en la lucha contra la violencia de género. Rev Metas de Enfermería. [revista en la internet]. 2009 [citado 2011 Jun 15]; 13 (1): 104-109. Disponible en: http://www.enfermeria21.com/images/PDF/actualidad131.pdf

42. Sans Mireia, Sellarés Jaume. Detección de la violencia de género en atención primaria. Rev. Med. Legal [revista en la internet]. 2010 [citado 2011 Jun 15]; 36 (3): 104-109. Disponible en: http://www.elsevier.es/es/revistas/revista-espa\%C3\%B1ola-medicina-legal-285/deteccionviolencia-genero-atencion-primaria-13189918-originales-2010

43. Snell JE, Rosenwald RJ, Robey A; The wife-beater's wife: a estudy of family interaction. Rev. Arch Gen Psychiatry, 1964; 11(2):107-113.

44. Suelves, Josep M.; Jané, Mireia; Plasència, Antoni. Violencia del compañero íntimo contra la mujer: una mirada desde la Salud Pública. Rev. Med. Legal [revista en la internet]. 2010 [citado 2011 Jun 15]; 36 (3): 98-103. Disponible en: http://www.elsevier.es/es/revistas/revistaespa\%C3\%B1ola-medicina-legal-285/violencia-compa\%C3\%B1ero-intimo-mujer-una-

mirada-salud-13189917-originales-2010

45. United Nations General Assembly. Declaration on the elimination of violence against women. Proceedings of the $85^{\text {th }}$ Plenary Meeting. Geneva, 20 de diciembre de 1993.

46. Velázquez, S.; "Extraños en la noche. La violencia sexual en la pareja", en Mabel Burín, Dio Bleichmar: Género, psicoanálisis, subjetividad. 1ae ed. Buenos Aires: Paidós; 1996. 47. VIII Congreso de Sociología. Marugán, B y Vega, C, El cuerpo contra-puesto. Discursos feministas sobre la violencia contra las mujeres. Salamanca. 2001.

48. Vives-Cases C, Caballero P, Álvarez-Dardet C. Análisis temporal de la violencia del compañero íntimo. Gac Sanit. 2004; 18:346-50.

49. Vives, C; Álvarez-Dardet, C; Caballero, P. Violencia del compañero íntimo en España. Rev. Gac Sanit. [Revista en la internet]. 2003 [citado 2011 Jul 27]; 17 (4): 268-74. Disponible en: $\quad$ http://www.elsevier.es/es/revistas/gaceta-sanitaria-138/violencia-compa\%C3\%B1erointimo-espa\%C3\%B1a-13051043-originales-2003

50. Vives-Cases C, Álvarez-Dardet C, Colomer C, Bernabeu MA. Una experiencia de Defensa de la salud sobre violencia de género. Gac Sanit. 2005;19:262-4.

51. Vives- Cases Carmen, Álvarez- Dardet Carlos, Carrasco-Portiño Mercedes, TorrubianoDomínguez Jordi. El impacto de la desigualdad de género en la violencia del compañero íntimo en España. Rev. Gac. San. [Revista en la internet]. 2007 [Citado 2011 Jun 15]; 21 (3): 242-6. Disponible en: http://www.elsevier.es/es/revistas/gaceta-sanitaria-138/el-impacto-desigualdadgenero-violencia-compa\%C3\%B1ero-intimo-13106808-originales-breves-2007 
52. Vives-Cases C, Álvarez-Dardet C, Gil-González D, Torrubiano-Domínguez J, Rohlfs I, Escribà-Agüir V. Perfil sociodemográfico de las mujeres afectadas por violencia del compañero íntimo en España. GacSanit. 2009; 23(5):410-4

53. Vives-Cases, Carmen; Gil-González, Diana; Carrasco-Portiño, Mercedes; ÁlvarezDardet, Carlos. Detección temprana de la violencia del compañero íntimo en el sector sanitario. ¿Una intervención basada en la evidencia? Rev. Med. Clin. (Barc) [Revista en la internet]. 2006 [Citado 2011 Jun 15]; 126 (3): 101-4. Disponible en: http://www.elsevier.es/es/revistas/medicinaclinica-2/deteccion-temprana-violencia-compa\%C3\%B1ero-intimo-sector-sanitario13083880-articulos-especiales-2006

54. Walker LE. The battered women. New York: Harper and row. 1979.

55. Watts C, Zimmerman C. Violence against women: global scope and magnitude. Lancet 2002;359:1232-7.

56. World Health Assembly (WHA). Prevention of violence: a public health priority (handbook of resolution). WHA, 25 de mayo de 1996 (Sixth plenary meeting, Committee B fourth report. 3rd ed).

57. World Health Organization. Estudio multipaís de la OMS sobre salud de la mujer y violencia doméstica contra la mujer Primeros resultados sobre prevalencia, eventos relativos a la salud y respuestas de las mujeres a dicha violencia. [monografía en Internet], Ginebra: Organización Mundial de la Salud; 2005. [citado 2011 Jul 31]; Disponible en: http://www.who.int/gender/violence.pdf

58. World Health Organization. World Report on violence Health, Ginebra. [Internet]. 2002 [citado 2011 Jun 15]; Disponible en: http://www5.who.int/violence injury prevention/download.cfm?id=0000000582.

59. XI Congreso de la Sociedad Española de Salud Pública y Administración sanitaria. Taller D: Grupo de género. Las desigualdades en salud según género. Rev. Gac. Sanit. [Revista en la internet]. 2001 [citado 2011 Jun 15]; 15 (Supl 3): 51-3. Disponible en: http://www.elsevier.es/es/revistas/gaceta-sanitaria-138/taller-d-grupo-genero-las-

desigualdades-salud-13021031-talleres-2001

60. Lorente Acosta, M.III Informa Anual del Observatorio Estatal de Violencia sobre la mujer.

Madrid: Ministerio de Sanidad, Política Social e lgualdad. 2010.

ISSN 1695-6141

๑ COPYRIGHT Servicio de Publicaciones - Universidad de Murcia 